\title{
Editorial: Applied Knowledge Management in an Institutional Context
}

\author{
Murali Raman* \\ Faculty of Management \\ Multimedia University, Malaysia \\ E-mail: murali.raman@mmu.edu.my \\ *Corresponding author
}

\section{Saravanan Muthaiyah}

Faculty of Management

Multimedia University, Malaysia

E-mail: saravanan.muthaiyah@mmu.edu.my

\begin{abstract}
This special issue aims to stimulate the investigation on the applications of KM principles, ideas and systems within an institutional context. Our editorial segment of this special edition proceeds as follows. The next section provides some stylized facts on $\mathrm{KM}$ and $\mathrm{KM}$ systems in general. Subsequently we examine two emerging areas in which KM in the context of its application is becoming vital - (i) in the area of ontology-based organizational memory and (ii) in the area of disaster management. We then provide a preview of the papers that were accepted in this special edition.
\end{abstract}

Keywords: Knowledge Management, Ontologies, Taxonomies, Organizational Memory, Emergency Response, Emergency Information Systems, Knowledge Evolution

\section{Biographical notes:}

Dr. Murali Raman received his PhD in Management Information Systems from the School of IS \& IT, Claremont Graduate University, USA. Dr. Murali is a Rhodes Scholar and a Fulbright Fellow. His other academic qualifications include an MBA from Imperial College of Science Technology and Medicine, London, an MSc in HRM from London School of Economics. Dr. Raman is currently an academician attached to Multimedia University Malaysia, where he teaches and conducts research in the area of Management Information Systems, Project Management and E-Business Models. He has published in more than fifty papers in International Journals and Conference proceedings. His corporate experience includes working with Maybank Malaysia (2 years) and Accenture Consulting (5years) before embarking on an academic path. He has consulted with numerous companies including several based in the USA (Claremont University Consortium), Ghana (GIMPA), Singapore (AsiaOne, Kepple bank), Australia (Monash Supply Chain and Logistics Center), and Malaysia (Panasonic, OUM, RHB Bank, Intan, MAKPEM, Malaysian Association of 
Social Workers, CDC Consulting among others). His research interests are mainly in the area of Knowledge Management Systems and its application in an institutional context.

Dr. Saravanan Muthaiyah received his Ph.D. in Information Technology from the Volgenau School of Information Technology and Engineering, Department of Computer Science, George Mason University, Fairfax, Virginia, USA. In his research he developed matching algorithms that reduced real-time human inputs for matching ontological data schemas in the Semantic Web domain. Dr. Saravanan Muthaiyah's matching algorithm had produced schema mappings which have improved matching efficiency form a 56\% accuracy to $92 \%$ accuracy. Dr. Muthaiyah is a Fulbright Fellow and was sponsored by the US Department of State during his doctoral studies in the US. He was also awarded several doctoral fellowships awards for his outstanding achievement during his graduate studies in George Mason University. Dr.Muthaiyah also holds a masters degree in Information Technology from the department of computer science and bachelors degree in Accounting and Finance. He is currently an academician in Multimedia University, Cyberjaya, Malaysia and also serves as deputy dean for the faculty of management. He also serves as a mini track chair for the HICSS 43 conference in Hawaii among being a associate member of other editorial boards of international journals and conferences. He has published more than fifty papers in international journals, books, workshops and conference proceedings. His corporate experience includes working with IBM World Trade Corporation, Intel (Research Fellow) and Arthur Anderson. He has consulted with numerous companies in USA, Malaysia, Singapore, Indonesia, Brunei, Thailand and Philippines.

\section{Introduction}

Davenport and Prusak (1998) define knowledge as an evolving mix of framed experience, values, contextual information, and expert insight that provides a framework for evaluating and incorporating new experiences and information. Knowledge often becomes embedded in documents or repositories, as well as in organizational routines, processes, practices, and norms. Knowledge is also about meaning, in the sense that it is context-specific. Jennex (2006) extends the concepts of context to also include associated culture that provides frameworks for understanding and using knowledge. A simpler definition of knowledge is that it is the how and why of something. Gaining knowledge is gaining insight into how and why things happen. To be useful, this knowledge must be framed within context and culture, providing the information and data needed; to explain how that knowledge is generated, what it means, and how it should be used.

Knowledge Management (KM henceforth) refers to ideas and principles that have been around for more than two decades, yet researchers worldwide continue working on this topic. In this special edition of the KM\&EL international journal, we would like to invite researchers working on $\mathrm{KM}$ issues to submit papers, under the broad theme of Applied KM. Specifically, the editor is keen in accepting papers pertaining to KM that 
are presented in an applied format, namely, papers that go beyond examining factors that impact KM adoption or its implications therein, in an organizational context.

This special issue aims to stimulate the investigation on the applications of KM principles, ideas and systems within an institutional context. Our editorial segment of this special edition proceeds as follows. The next section provides some stylized facts on KM and KM systems in general. Subsequently we examine two emerging areas in which KM in the context of its application is becoming vital - (i) in the area of ontology-based organizational memory and (ii) in the area of disaster management. We then provide a preview of the papers that were accepted in this special edition.

\section{Knowledge Management (KM) and KM Systems}

A single definition of KM does not exist (Alavi \& Leidner, 2001; Nonaka \& Takeuchi, 1995; Nonaka \& Konno., 1998). However, Jennex (2005) used an expert panel to generate a composite definition of KM as the practice of selectively applying knowledge from previous experiences of decision-making to current and future decision making activities with the express purpose of improving the organization's effectiveness. Alavi and Leidner (2001, p. 114) defined a KM System, KMS, as "IT (Information Technology)-based systems developed to support and enhance the organizational processes of knowledge creation, storage/retrieval, transfer, and application." They observed that not all KM initiatives will implement an IT solution, but they support IT as an enabler of KM. Gupta and Sharma (2004) classify KMSs into seven major categories, and this includes among others ontologies.

The purpose of implementing KMS in organizations varies. Von Krogh (1998) takes a business perspective, stating that KMS help increase competitiveness. Hackbarth (1998) suggests that KMS lead to greater innovation and responsiveness. Davenport and Prusak (1998) provide three reasons why KMS are implemented in organizations: (i) to enhance visibility of knowledge in organizations through the use of maps, hypertexts, yellow pages; directories, etc., (ii) to build a knowledge sharing culture, i.e., create avenues for employees to share knowledge, and (iii) to develop a knowledge infrastructure, not confined to technology solely, but to create an environment that permits collaborative work. Work by Hackbarth (1998) and Davenport and Prusak (1998) imply that KMS can support an organization in planning for and dealing with crises.

\section{Emerging Area 1 - Ontology based Organizational Memory}

An ontology is a formal specification of a domain of knowledge and provides a shared understanding of that domain of knowledge (Gruber, 1993). It describes concepts and relationships of a knowledgebase that makes it machine processable and understandable. In other words, it provides a common platform for software robots (i.e. softbots) to decipher data concepts and relationships explicitly. In terms of formality, there is a spectrum of formal specifications (see Figure 1). At one extreme (the left side of the spectrum), there are lightweight ontologies that consist of terms only, and at the other end (the right side of the spectrum), there are more formal ontologies and inferences. 


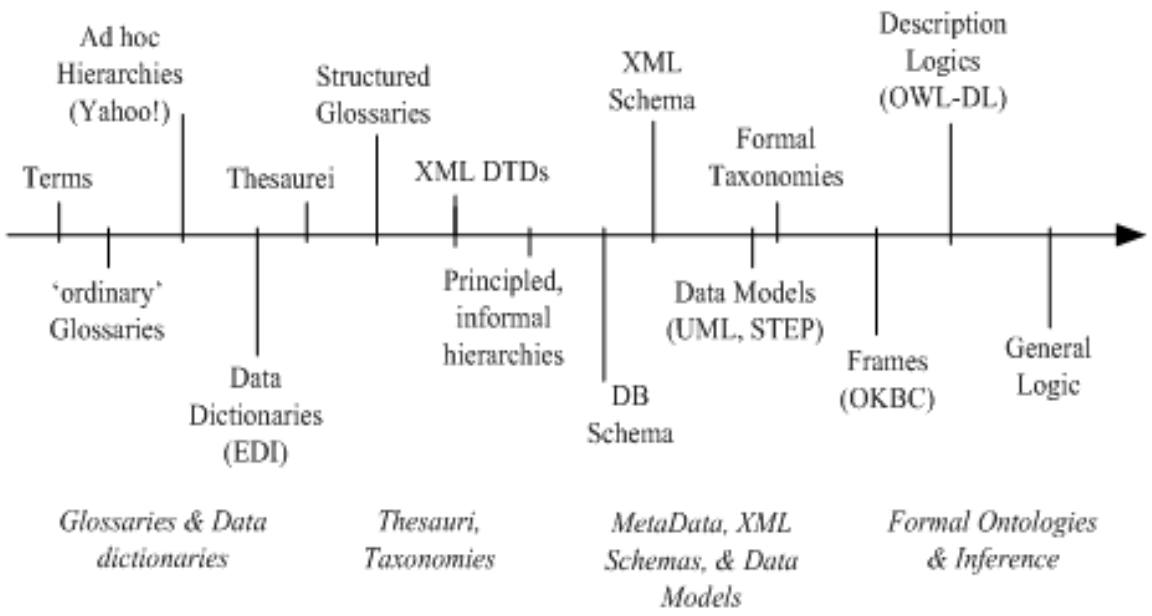

Figure 1. A spectrum of Ontology

There are various definitions for ontologies such as "a specification of conceptualization" and "an abstract model of how a domain is perceived". The former implies that ontologies provide explicit names and definitions for the concepts and relationships. The latter shows how an explicit existence of such shared perspectives makes it possible for both humans and softbots to collaborate by ensuring that everyone makes the same distinctions and use the same terms in the same context (Fox et al., 1998).

This is possible with the advent of data interchange formats such as N-Triples, RDF (Resource Description Framework), Turtle (Terse RDF Triple Language) and OWL (Web Ontology Language). The key element here is to remove any level of ambiguity that may arise in defining data concepts. If ambiguity exists, then a common level of understanding cannot be achieved and this would in turn create different variations in knowledge. This will in- turn will make it impossible for the KMS to produce consistent knowledge. Inconsistency in knowledge may result in inaccurate decisions. The crux of an organizational memory is the ability of it to produce efficient, timely and accurate decisions somewhat like a functioning human brain. At the same time it should also be equipped with the power to "think" and "reason" just like how a human brain would. In an institutional context KMSs are built on taxonomies, data schema, metadata, XML schemas, data models, OWL-DL (web ontology language description logic), frames (OKBC), data models, logic and formal ontologies that provide the KMSs the power to think and reason which is often referred to as inference analysis. The organizational memory is the core application of a KMS. Institutions today rely heavily upon their KMS to be more competitive. The efficiency in inference analysis and reasoning clearly distinguishes one KMS from the other. This would provide institutions the additional wealth of knowledge that others could lack, which is really significant in such a competitive world.

Ontologies are useful for the following:

i. Retrieving the appropriate information from documents by providing a structure to annotate the contents of a document with semantic information [Alani et al., 2003; Gibbins et al., 2003]. 
ii. Integrating the information from various sources by providing a structure for its organisation and facilitating the exchange of data, knowledge and models [Anonymous, 2000; Anonymous, 2002].

iii. Ensuring consistency and correctness by formulating constraints on the content of information [Anonymous, 2002].

iv. Creating libraries of interchangeable and reusable models [Anonymous, 2000; Anonymous, 2002].

v. Supporting inference to derive additional knowledge from a set of facts [G'omez et al., 2001; Anonymous, 1999].

Ontologies provide the platform for organizations today to build a knowledge base. Knowledge on the other hand is never static and evolves constantly. KMSs today must be enabled with the ability to capture knowledge evolution and patterns of versioning. Our recent work in this area has been receiving a lot of attention and we feel is the way forward for all KMSs.

\section{Emerging Area 2 -Crisis Response}

Crisis Response Systems are used by organizations to assist in responding to a crisis situation. These systems support communications, data gathering and analysis, and decision-making. Crisis Response Systems are rarely used but when needed, must function well and without fail. Designing and building these systems requires designers to anticipate what will be needed, what resources will be available, and how conditions will differ from normal. A standard model for a Crisis Response System is from Bellardo, Karwan, and Wallace (1984) and identifies the components as including a database, data analysis capability, normative models, and an interface. This model is only somewhat useful as it fails to address issues such as how the Crisis Response System fits into the overall crisis response plan, Crisis Response System infrastructure, multiple organization spanning, knowledge from past emergencies, and integrating multiple systems. Additionally, many organizations do not address the need for a Crisis Response System until a crisis happens, and then, only for a few months until something more pressing comes up (Jennex, 2003). The result is that many organizations have a Crisis Response System that may not be adequate.

Prior to the establishment of the Homeland Security Department, the task of managing information pertaining to crisis situations and crisis management in the United States was under the jurisdiction of the Office of Emergency response (OEP) (Turoff, 1972). The information requirements for the OEP were largely handled by a group of consultants from both business and academia. Over time, the OEP recognized that a system that could provide timely and relevant information to crisis responders was needed (Turoff, 1972). In 1970, twenty-five people working on crisis response were able to collaborate via a computerized Delphi system (Turoff, 1972). Computerized Delphi techniques can be administered via the web today (see for example Turoff, 2003 and Turoff and Hiltz, 1995).

In 1971, the OEP was assigned the task of monitoring a new form of crisis called the "Wage Price Freeze" (Turoff et al., 2004). This new role for the OEP included among others, to "monitor nationwide compliance, examine and determine requests for exemptions and prosecute violations" (p.5) in relation to wage and price changes in the economy. This led to the advent of a flexible system called the Emergency Management 
Information System and Reference Index (EMISARI). EMISARI was a system designed to facilitate effective communication between people involved in monitoring the Wage Price Freeze situation. The system was designed to integrate people and data into a common platform that could be updated regularly by people who were non-technical administrators (Turoff et al., 2004). The EMISARI system was flexible and enabled several hundreds of people to collaborate in responding to a crisis (see for example Rice 1987, 1990 and Turoff, 2002).

Jennex (2004) classifies crisis management information systems as Emergency Information Systems (EIS). He defines an EIS as any system that is used "by organizations to assist in responding to a crisis or disaster situation" (p. 2148). Jennex further adds that an EIS should be designed to: support communication during crisis response; enable data and gathering analysis; and support decision-making. Lee and Bui (2000) documented vital observation with the use of EIS during the massive earthquake that hit Kobe, Japan, several years ago.

Key lessons for crisis management system designers based on Lee and Bui's work are as follows. Relevant information should be included in the crisis response system prior to the actual crisis situation. This is to ensure that crisis responders have sufficient information to guide the decision-making processes in responding to a crisis. Lee and Bui (2000) imply that the task of gathering relevant information to support crisis response should be incorporated into part of the crisis response strategic initiative. Information from prior experiences should become part of the crisis management system. The system should somehow be able to capture both tacit and explicit knowledge about how prior crisis situations were dealt with. Lessons, which are learned, can be used to guide future action. Lee and Bui (2000) in this regard imply that the design of any crisis response system should support some form of organizational memory component.

In addition to designing relevant systems features to support crisis planning and response, researchers suggest that successful implementation of any crisis management system is contingent on how well people are trained to use such systems (Patton \& Flin, 1999; Turoff, 1972; Lee \& Bui, 2000). Patton and Flin, for instance, suggest that crisis management systems be incorporated into crisis response related activities such as training, simulations, drills, and evacuation exercises. Turoff (1992) states that crisis management systems that are not normally used will not be used when an actual crisis situation occurs.

The majority of post 9/11 literature on crisis management is confined within the realm of commercial entities (Braveman, 2003). Developments within the domain of crisis management information systems have accelerated over the past few years, particularly after the 9/11 events (Campbell et al., 2004). The authors accurately mention that issues such as resources, expertise, and personnel should be addressed at the onset, prior to designing crisis management systems within the context of local and state level communities. They call for development of "a generic set of requirements" (p.2) that can be used by both the state and local authorities to support crisis planning and response. The researchers however do not base their study on any particular theoretical foundations. Campbell and associates (2004) examine the effect of asynchronous negotiation given "a structured task and a specified negotiation sequence" (p.3), in the context of crisis responders. 


\section{Preview of Papers}

Four papers were accepted under this special edition. In the first paper, Chen examines an important question namely, why do organizations face difficulties in successfully manipulating knowledge created/generated in an institutional context. Using the KMOntology relationship, Chen offers valuable insights on how KM practices can benefit from ideas inherent in Lean Sigma best practices. Specifically, by applying case study methodology Chen suggests that to maximize the value potential of KM practices in the context of enhancing knowledge sharing, the ideals from KM and lean Sigma can be blended.

The second paper focuses directly on applying KM to a learning context. Martin offers ideas on how semantic networks as an instantiation of KM systems, can be used to cultivate greater learning outcomes, relative to traditional open/distance learning tools and practices. He developed a tool based on wiki engine called WebKB-2 to create the semantic networks, in the context of gauging learning amongst students. Surveys were then administered to students to ascertain if WebKB-2 was effective to support learning processes within an institutional setting. The author states that by combining wiki based engines with the WebKB-2 platform, structured learning via knowledge sharing can be enhanced.

The third paper, while it does not exactly relate to KM application, examines a useful issue. Turner, Biros and Moseley extend the notion of task-technology-fit (TTF) to the domain of KM systems and assert that the TTF power as a predictive model can be further examined by incorporating other elements to fit with a KM applied context. This they argue can add greater value to future work that measure if indeed KM systems when applied within an institutional context, can add value to end users and organizations alike.

The final paper examines a real world knowledge sharing idea, presented in the context of informal communities in Cyberjaya, Malaysia. Azudin, Ismail and Taherali examine the role of culture and how this impacts knowledge sharing amongst informal communities in Cyberjaya, Malaysia. The authors suggest that informal communities e.g. lunch and other lesser than formal settings are vital events that can foster knowledge sharing amongst communities in a given locality.

\section{Conclusion}

Organizations today face difficulties in successfully manipulating knowledge generated in an institutional context due to reasons presented in Chen's paper as well as due to lack of contextual understanding of metadata that has been captured in their respective KMSs. This, bring us back to the discussion on the lack of a common level of understanding discussed previously. The second paper highlights applying KM to a learning context which again reflects the importance of our discussion on knowledge evolution. Semantic Web technologies have been very successful in this domain especially in producing learning algorithms and inference analysis via description logic tools. The notion of tasktechnology-fit (TTF) then becomes evident with real world KMSs especially in the domain of emergency response systems as discussed earlier. Lastly the concept of collaborative knowledge leads to KMSs that are built for informal knowledge sharing among individuals. This could also be extended to systems that are built around social networking portals such as Facebook so as to develop informal and static based community knowledge base. In conclusion this editorial segment has presented the role and relevance of KMS to support organizational memory and crisis response efforts. 
Researchers are encouraged to examine design and development issues of KMSs in support of other critical arrears such as defense and health for future research.

\section{References}

1 Alani, H., Kim, S., Millard, D., Weal, M., Hall, W., Lewis, P., \& Shadbot. N. (2003). Automatic ontology based knowledge extraction from web documents. IEEE Intelligent Systems, 18(1), 14-21.

2 Alavi, M. \& Leidner, D.E. (2001). Review: Knowledge Management and Knowledge Management Systems: Conceptual Foundations and Research Issues. MIS Quarterly, 25(1), 107-136.

3 Anonymous (1999). On-To-Knowledge. IST project IST-1999-10132 On-ToKnowledge, http://www.ontoknowledge.org/.

4 Anonymous (2000). IST project IST-2000-28384 Agentcities, http://www.agenticities.net/.

5 Anonymous (2002). OntoWeb. IST project IST-2000-29243 OntoWeb, http://www.ontoweb.org.

6 Anonymous (2005). Public Safety Canada, http://www.publicsafety.gc.ca/media/nr/2005/nr20051117-en.asp.

7 Bellardo, S., Karwan, K.R., \& Wallace, W.A. (1984). Managing the Response to Disasters Using Microcomputers. Interfaces, 14(2), 29-39.

8 Braverman, M. (2003). Managing the human impact of crisis. Risk Management, 50(5), 10-19.

9 Campbell, C. L., DeWalle, B. V., Turoff, M., \& Deek, F. P. (2004). A Research Design for Asynchronous Negotiation of Software Requirements for an Emergency Response Information System. Paper presented at the Americas Conference on Information Systems, New York City.

10 Davenport, T.H. \& Prusak, L. (1998). Working Knowledge. Harvard Business School Press.

11 Fox, M. S., Barbuceanu, M., Gruninger, M., \& Lin, J. (1998). An organization ontology for enterprise modelling. In M. Prietula, K. Carley \& L. Gasser (Eds.), Simulating organizations: Computational models of institutions and groups (pp. 131-152): AAAI Press.

12 Gruber, T.R. (1993). Toward Principles of Design Ontologies Used for Knowledge Sharing. paper presented at the International Workshop on Formal Ontology, Padova, Italy.

13 Gibbins, N., Harris, S., \& Shadbolt, N. (2003). Agent-based semantic web services. Paper presented at the Twelfth International World Wide Web Conference (WWW2003), Budapest, Hungary, ACM Press.

14 Gupta, J. D., \& Sharma, S. K. (2004). Creating Knowledge Based Organizations: IDEA Group Publishing.

15 Hackbarth, G. (1998). The Impact of Organizational Memory on IT Systems, paper presented at the Fourth Americas Conference on Information Systems.

16 Jennex, M.E. (2003). Information Security in the Era of Terrorist Attacks. Information Resource Management Association International Conference Panel.

17 Jennex, M.E. (2004). Emergency Response Systems: The Utility Y2K Experience. Journal of IT Theory and Application (JITTA), 6(3), 85-102. 
18 Jennex, M.E. (2005). Knowledge Management Systems. International Journal of Knowledge Management, 1(2), 1-4.

19 Jennex, M.E. \& Olfman, L. (2006). A Model of Knowledge Management Success. International Journal of Knowledge Management, 2(3), 51-68.

20 Lee, J., \& Bui, T. (2000). A Template-based Methodology for Disaster Management Information Systems. Paper presented at the Hawaii International Conference on Systems Science, Hawaii.

21 Nonaka, I., \& Takeuchi, H. (1995). The Knowledge-Creating Company? New York: Oxford University Press.

22 Nonaka, I., \& Konno, N. (1998). The concept of "ba": Building a foundation for knowledge creation. California Management Review, 40(3), 40-54.

23 Patton, D., \& Flin, R. (1999). Disaster Stress:An Emergency Management Perspective. Disaster Prevention and Management, 8(4), 261-267.

24 Rice, R. E. (1987). Information, Computer Mediates Communications and Organizational Innovation. Journal of Communication, 37(4), 65-94.

25 Rice, R. E. (1990). From Adversity to Diversity: Applications for Communication Technology to Crisis Management. Advances in Telecommunications Management, 3, 91-112.

26 Turoff, M. (1972). Delphi Conferencing: Computer Based Conferencing with Anonymity. Journal of Technological Forecasting and Social Change, 3(2), 159204.

27 Turoff, M. (2002). Past and Future Emergency Response Emergency Response Information Systems. Communications of the ACM, 45(4), 38-43.

28 Turoff, M., \& Hiltz, S. R. (1995). Computer Based Delphi Processes. In M. Adler \& E. Ziglio (Eds.), Gazing Into the Oracle: The Delphi Method and Its Application to Social Policy and Public Health. London: Kingsley Publishers, 56-88

29 Turoff, M., Chumer, M., Van de Walle, B., \& Yao, X. (2004). The Design of a Dynamic Emergency Response Management Information Systems (DERMIS). Journal of Information Technology Theory and Application, 5(4), 1-35.

30 Von Krogh, G. (1998). Care in Knowledge Creation. California Management Review, 40(3), 133-153. 